\title{
Effectiveness of the Treatment with Botulinum Toxin type A (BTX-A) in the Management of the Spasticity in Patients with Amyotrophic Lateral Sclerosis (ALS)
}

\author{
Marvulli R. ${ }^{1,2,3, *}$, Megna M. ${ }^{1,2,3}$, Romanelli E. ${ }^{1,3}$, Mastromauro L. ${ }^{1,3}$, Conte E. ${ }^{2}$, Lancioni G. ${ }^{1}$, Dargenio M. ${ }^{1}$, \\ De Venuto G. ${ }^{1}$, Gallo G.A. ${ }^{1}$, Lerario R. ${ }^{1}$, Fiore P. ${ }^{1,3}$ and Ianieri G. ${ }^{1,2,3}$ \\ ${ }^{1}$ Department of Basic Sciences, Neuroscience and Sense Organs, University of Bari "Aldo Moro", Bari, Italy; ${ }^{2}$ School \\ of Advanced International Studies on Applied Theoretical and Non Linear Methodologies of Physics, Bari, Italy and \\ ${ }^{3}$ Department of Physical Medicine and Rehabilitation, University of Bari “Aldo Moro", Bari, Italy
}

A R T I C L E H IS T O R Y

Received: June 20, 2016 Revised: August 02,2016 Accepted: August 05, 2016 DOI: $10.2174 / 22127070036661608101438$ 54

\author{
Ar
}

Abstract: Amyotrophic Lateral Sclerosis (ALS) is an unknown pathogenesis progressive neurodegenerative disease of the central nervous system that leads to death within 1-5 years. Clinically, it is often possible to find at the level of the lower limbs some clinical manifestations of damage of the I motor neuron with spastic paralysis, iperflexia and clonus, with impairment of the ability of patients' deambulation and their management of the activities of daily living (such as personal hygiene or dressing). So, the first therapeutic approach in these patients are antispasmodic drugs orally and after Botulinum toxin type A injection (BTX-A). Aim of this study is to demonstrate the efficacy of BTX-A in patients with ALS and spasticity of lower limbs no responder to the treatment with oral antispastic drugs with no adverse events. We enrolled 5 patients ( 3 female and 2 male); they were evaluated at baseline (T0, before BTXA treatment), and over the following three months with three follow-up visits (T1 30 days after the infiltration, T2 60 days after infiltration and T3 90 days after infiltration) with myometric measure of tone, Modified Ashworth Scale, Barthel Index, Adductor Tone Rating Scale and Hygiene Score. We treated the adductor muscles (AM) of patients with incobotulinum toxin type A (Xeomin ${ }^{\circledR}$, Merz) with ultrasound guide. We obtained an improvement of spasticity with miometric measurement, Modified Ashworth Scale, Barthel Index, Adductor Tone Rating Scale and Hygiene Score for 90 days after injection $(\mathrm{p}<0,05)$. Our preliminary study shows the possibility to use BTX-A in the treatment of the spasticity in patients with ALS no responders to oral antispastic drugs, with no side effects.

Keywords: Amyotrophic Lateral Sclerosis, spasticity, botulinum toxin type A, activities of daily living, physiotherapy, oral antispastic drugs

\section{INTRODUCTION}

Amyotrophic Lateral Sclerosis (ALS) is a progressive neurodegenerative disease of the central nervous system with an unknown pathogenesis[1]. This devastating neurodegenerative disease usually leads to death within 1-5 years period of diagnosis [2,3]. Clinically ALS is characterized by a gradually involvement of the motor neurons both upper (I motor neuron), localized at the level of the cerebral cortex (UMN), and lower (II motor neuron), situated in the brainstem and spinal cord (LMN). Diagnostic criteria for ALS rely on clinical assessment of upper and lower motor neuron deficits in multiple body regions together with a history of progres

*Address correspondence to this author at the Department of Basic Sciences, Neuroscience and Sense Organs, University of Bari "Aldo Moro", Bari, Italy; E-mail: ricmarv81@ hotmail.it sion of symptoms, and also on para-clinical observation of LMN by electromyography [4]. No para-clinical assessment of UMN involvement has reached enough validity to be included in diagnostic criteria. In the past decade nonconventional magnetic resonance techniques have been shown to be sensitive to detect UMN involvement in the ALS disease process, namely: resting-state connectivity measured with functional MRI, cortical thickness measured by high-resolution imaging, diffusion tensor imaging (DTI) metrics such as fractional anisotropy and radial diffusivity, and more recently magnetic resonance spectroscopy (MRS) measures of gamma-aminobutyric acid concentration[5]. An additional symptom shared by ALS is the occurrence of neuroinflammation and infiltration of T-lymphocytes in some areas of the CNS or spinal cord $[6,7,8]$; in fact the number of regulatory $\mathrm{T}$-lymphocytes correlates inversely with disease 
progression $[9,10]$. Clinically, in the majority of cases, it shows with signs of damage of II motor neuron with flabby paralysis (sign of the claw hand), areflexia, muscular atrophy and muscle fasciculations, giving rise to muscular weakness and atrophy; if it concerns the cranial nerves appears dysphagia, dysarthria and dysphonia [11]. This loss of motor neurons leads to fatal paralysis and death and is the most frequent disease of motor neurons [2,12]. It is often possible to find at the level of the lower limbs some clinical manifestations of damage of the I motor neuron with spastic paralysis, iperflexia and clonus. These clinical symptoms can jeopardize both the ability of patients' deambulation and their management of the activities of daily living (ADL) such as personal hygiene or dressing $[12,13]$.

Spasticity sometimes appears as symptom of ALS. Muscles are contracted and they are opposed to stretching by fixing the joints in forced positions, resulting in joint pain. The first therapeutic approach in these cases is the therapy with antispasmodic drugs orally (BACLOFEN) [14,15]. In some cases spasticity can also be resistant to these drugs and be a source of very intense pain or fix the limbs in positions that hinder, for example, the hygiene and care of the person. It may be necessary to use Botulinum toxin type A (BTX-A), injected into the muscles, to determine paralysis reducing spasticity. To treat therefore the symptom spasticity can be useful to improve the quality of life of the patients.

The aim of this study is to demonstrate the efficacy of BTX-A in patients with ALS and spasticity of lower limbs no responder to the treatment with oral antispastic drugs with no adverse events.

\section{MATERIALS AND METHODS}

\section{Study Population and Inclusion Criteria}

We enrolled 10 patients ( 6 female and 4 male) with mean age of $49,4 \pm$ SD 3,8 .

Inclusion criteria were: 1- diagnosis of probable or definite ALS based on revised El Escorial Criteria (Brooks 2000); 2- presence of spasticity of lower limbs that determined a progressive disability in the Activities of Daily Living (ADL); 3- previous use of oral antispastic drugs (Liore$\mathrm{sal} \circledast 75 \mathrm{mg} / \mathrm{die})$; 4- inefficacy of previous therapies or appearance of side effects such as cough, dyspnea and asthenia that had led to the interruption of the treatment; 5- absence of antibiotic therapy during the week preceding the treatment; 6- availability of the patient to undergo a continuing and intensive physiotherapy, every day for the first 30 days and then tri-weekly for the following two months, after the treatment; 7- absence of fibrotic degeneration of the spastic muscles, evaluated by ultrasounds.

All patients signed written informed consent.

Study was conducted between January and May 2016.

\section{Methods}

Patients were evaluated at baseline (T0, before BTX-A treatment), and over the following three months with three follow-up visits (T1 30 days after the infiltration, T2 60 days after infiltration and T390 days after infiltration).

\section{At baseline were performed:}

1- neurological visit by a neurologist, expert in motor neuron diseases. The evaluation included a standard neurological exam and the functional assessment through the Amyotrophic Lateral Sclerosis- Functional Rating Scale revised (ALSFRS-r) (Cedarbaum 1999);

2- the ultrasonography study of the muscles of interest to exclude the presence of fibrotic degeneration.

At baseline and during the follow-up visits patients underwent a physiatric examination with the use of myometric measure to evaluate muscle tone, elasticity and stiffness and the following scales: Modified Ashworth Scale, Barthel Index, Adductor Tone Rating Scale, and Hygiene Score $[16,17]$.

After the treatment patients started an intensive rehabilitation program consisted in muscular stretching, functional rehabilitation, active and passive mobilization, training of the step, exercises for the perception of herself, advising not to exceed the hard work threshold[18]. This program was carried out every day for the first 30 days and then tri-weekly for the following two months.

At the physiatric examination patients showed: independent erected standing possible only with bilateral support and for few minutes, deambulation possible with double support for a short time, by little steps and of precautionary type. It was present spastic paraparesis. Our patients complained both a difficulty in washing and dressing themself because of an excessive tone at the level of the adductor magnus (AM) bilaterally that determined a crossing of the lower limbs and a difficulty in the deambulation.

We treated the adductor muscles (AM) of patients with incobotulinum toxin type A (Xeomin ${ }^{\circledR}$, Merz); mean dose was 78,33 $\pm 9,18$; we injected BTX-A with ultrasound guide.

\section{Statistical Analysis}

Data were expressed as mean (standard deviation). The TWO - WAY ANOVA method was used to compare statistical differences of myometric measures and scales' value between baseline and follow-up.

\section{RESULTS}

We obtained the following results:

1- The mean values of muscle tone measured by myoton at the beginning of the study ( $\mathrm{t} 0 \mathrm{AM} \mathrm{dx}=18,45 \pm 1,23$, t0 AM $\mathrm{sn}=17,99 \pm 1,11)$ decreased in a statistically significant at $\mathrm{t} 1(\mathrm{AM} \mathrm{dx}=15,30 \pm 1,16, \mathrm{AM} \mathrm{sn}=15,32 \pm 1,12)$ and t2 (AM dx $=15,45 \pm 1,21, \quad A M \quad s n=15,99 \pm 1,22$ ), with $\mathrm{p}<0,05$. At $\mathrm{t} 3$, instead, data was not statistically significant if compared to the initial value $(\mathrm{AM} \mathrm{dx}=17,67 \pm 1,21$, $\mathrm{AM} \mathrm{sn}=17,11 \pm 1,02$ ), with $\mathrm{p}<0,05$. (Fig. 1)

2- Values of MAS (to $\mathrm{AM} \mathrm{dx}=3 \pm 1,1, \mathrm{AM} \mathrm{sn}=3 \pm 1,1$ ) also followed this trend, with statistic significance at t1 (AM $\mathrm{dx}=1,4 \pm 0,8, \mathrm{AM} \mathrm{sn}=1,3 \pm 1,0)$ and $\mathrm{t} 2(\mathrm{AM} \mathrm{dx}=1,4 \pm 0,8$, $\mathrm{AM} \quad \mathrm{sn}=1,3 \pm 0,8)$ and no significance at $\mathrm{t} 3$ (AM $d x=2,6 \pm 1,1, A M s n=2,7 \pm 0,6$ ), with $p<0,05$. (Fig. 2).

3- The baseline mean value of Barthel Index (to $=31 \pm 9,1)$ increased in a statistically significant way at $\mathrm{t} 1(49 \pm 4,1)$ 
and t2 $(46 \pm 4,8)$, with $\mathrm{p}<0,05$. At $\mathrm{t} 3$, instead, value $(34 \pm 5,4)$ was not statistically significant if compared to the initial value, with $p<0,05$. (Fig. 3)

4- The baseline mean value of Adductor tone rating scale (to $=3 \pm 1,1)$ decreased in a statistically significant way at $\mathrm{t} 1(1,3 \pm 0,8)$ and $\mathrm{t} 2(1,4 \pm 0,8)$, with $\mathrm{p}<0,05$. At $\mathrm{t} 3$, instead, value $(3 \pm 1,1)$ was not statistically significant if compared to the initial value, with $\mathrm{p}<0,05$. (Fig.4)

5- The baseline mean value of with Hygienic score $($ to $=3,2 \pm 1,3)$ decreased in a statistically significant way at $\mathrm{t} 1(1,3 \pm 0,4)$ and $\mathrm{t} 2(1,4 \pm 0,6)$, with $\mathrm{p}<0,05$. At $\mathrm{t} 3$, instead, value $(3,2 \pm 0,5)$ was not statistically significant if compared to the initial value, with $p<0,05$. (Fig.5)

\section{DISCUSSION}

Spasticity often is a typical symptom of ALS. The first therapeutic approach is the therapy with antispasmodic drugs orally; spasticity can often be resistant to these drugs with very intense pain or fixing the limbs in positions that hinder, for example, the hygiene and care of the person. It may be necessary to use BTX-A, injected into the muscles, to deter- mine paralysis reducing spasticity. This study shows that BTX-A, normally used treating sialorrhea[19], associated with moderate physiotherapy[20], can be helpful in improving spasticity of patients with ALS for three months at least. The treatment is safe, because we had no side effects such as general weakness and/or dysphagia. Our study shows that combined therapy BTX-A/FKT in patients with ALS can improve the rheological parameters of the spastic muscle tissue (see Fig.1 and 2); in this way it makes easier exercise with the slowing of the decay of motor skills, acting both cardio-respiratory efficiency both on complications from reduced joint mobility. Clinical benefits obtained from this activity relate to the physical efficiency, mood and quality of sleep (all 5 patients reported an increase in the number of hours of sleep). The activity of stretches and exercises of joint range prevent muscle contractures and retractions of connective tissues favored by immobility, as well as help to contain the resulting pain syndromes. The joint mobilization should be practiced by the patient or caregiver daily. The study also proves that the specific action on the AM muscles supports their passive motion (see Fig. 3). Patients have a better personal hygiene and a better personal autonomy (see

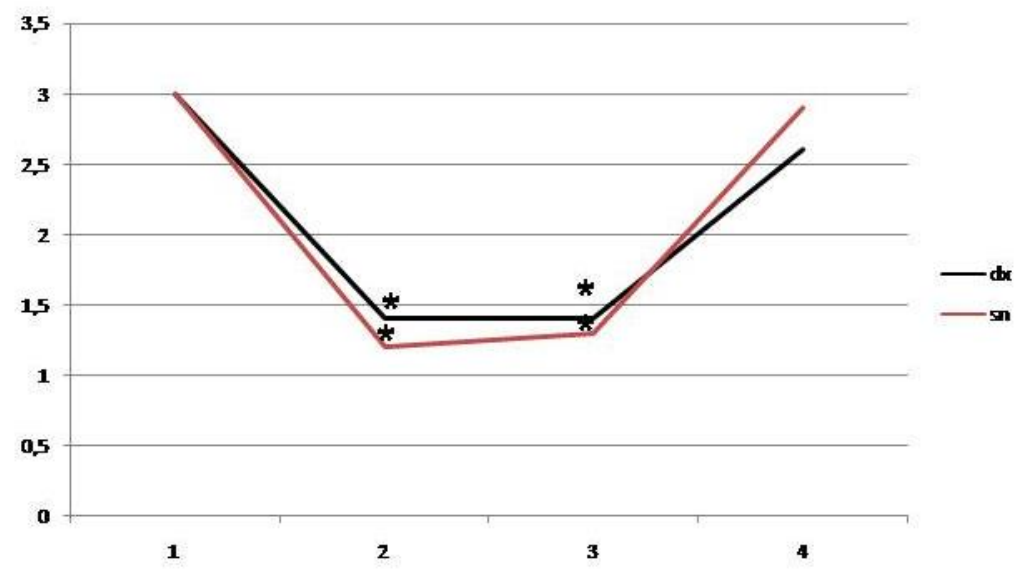

Fig. (1). Miometric tone modification of Adductor Magnus right and left; we demonstrated a statistical difference reduction of tone until 90 days after injection $(\mathrm{p}<0,05)$.

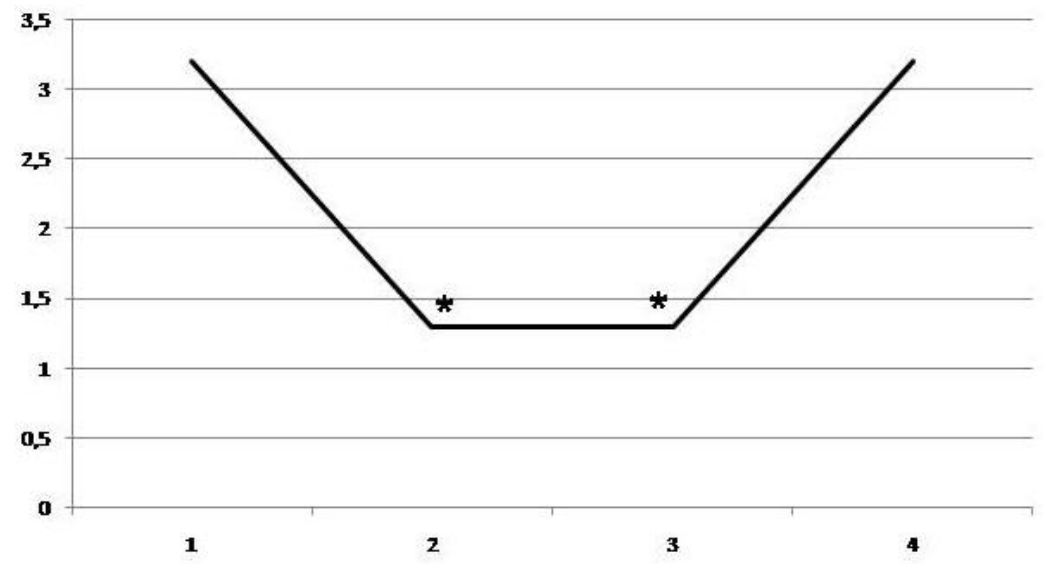

Fig. (2). MAS modification of Adductor Magnus right and left; we demonstrated a statistical difference reduction until 90 days after injection $(\mathrm{p}<0,05)$. 


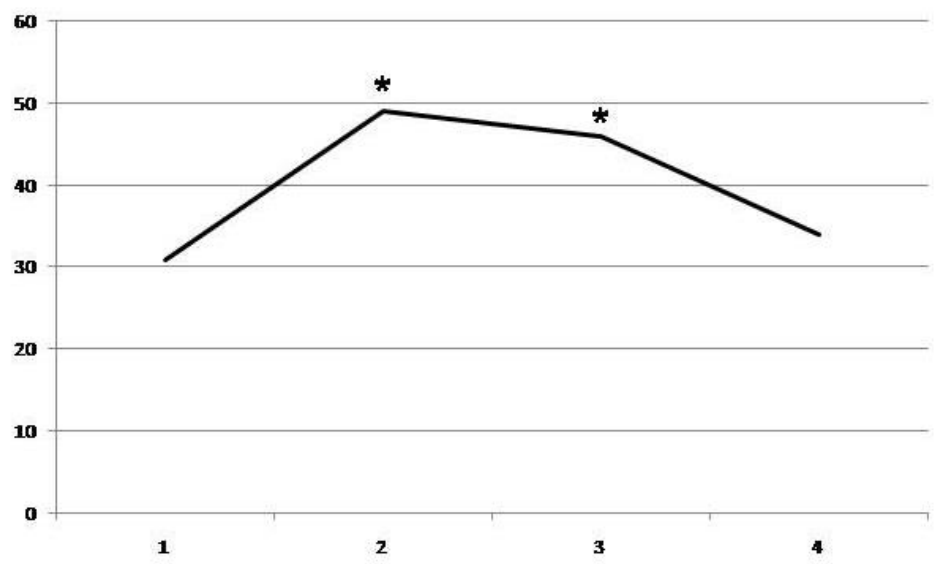

Fig. (3). Barthel Index modification of patients; in the Fig. (we observed a statistical improvement of value until 90 days after injection (p<0,05).

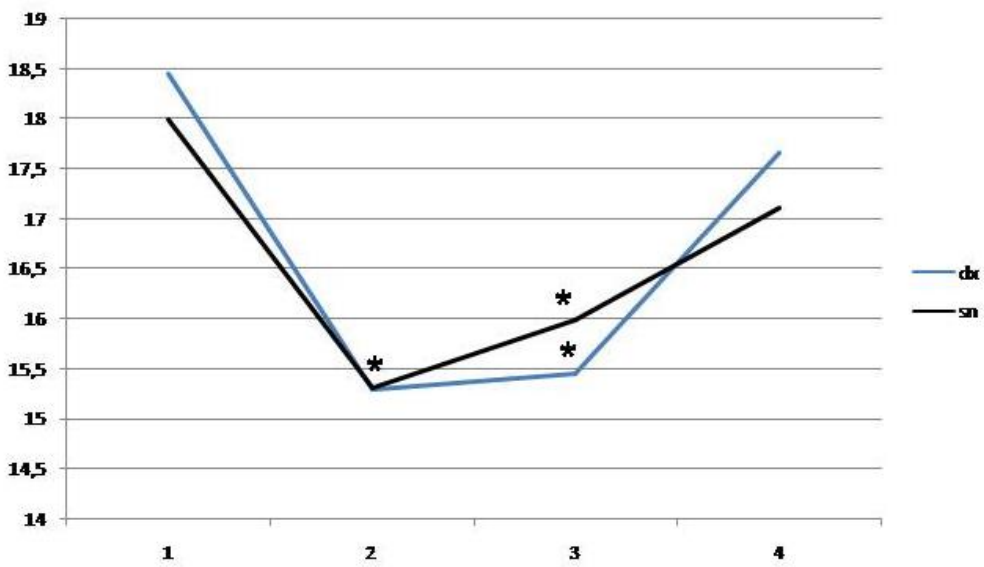

Fig. (4). Adductor tone rating scale modification of right and left muscles; we observed a statistical reduction of value until 90 days after injection ( $<<0,05$ ).

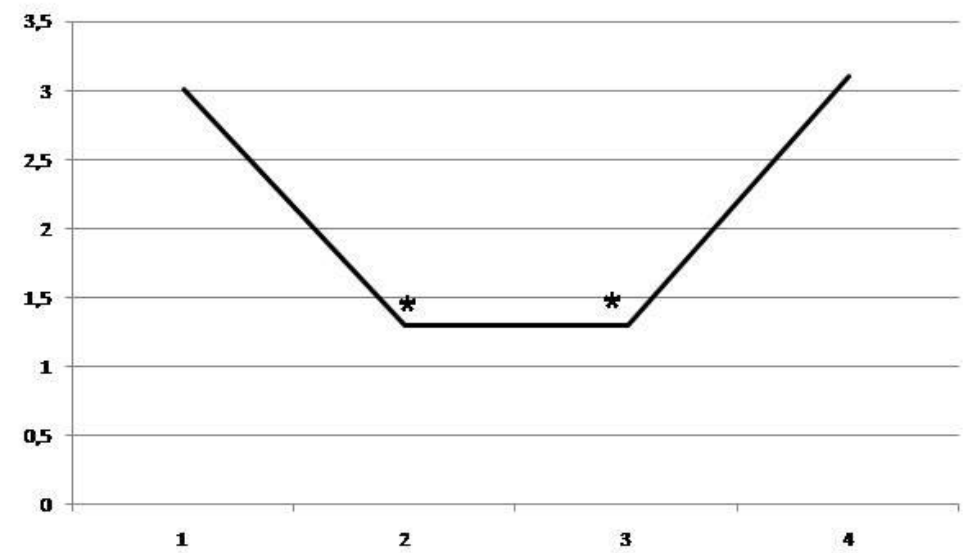

Fig. (5). Hygienic score modification; we demonstrated a statistical reduction until 90 days after injection ( $<<0,05)$.

fig. 4 and 5), for which they can be made easier some maneuvers, such as the catheterization or the hygiene of the perineal areas, which reduce the risk of infection of urinary tract, often leading cause of death in these patients. Combination therapy BTX-A/ physiotherapy has also shown efficacy in the onset of fatigue. Fatigue recognizes multifactorial etiology[21], being able to achieve the progressive deficit of strength and muscle tone (which favors a mechanical strain on joints and bone segments) and / or the onset of contractures $\backslash$ muscle spasms from spastic hypertonia that determine the appearance of degenerative lesions of the joints and connective tissue retraction from prolonged immobility and alterations in the microcirculation. So the reduction of muscle tone and easier mobilization of skeletal segments of a limb, by mechanisms still poorly understood, may be help in reducing the onset of fatigue. 
BTX-A and rehabilitation allowed a reduction of muscular tone (analyzed both in subjective ways with the Modified Ashworth Scale or with the objective appraisal of miometric measure) and an improvement of the muscular elasticity and stiffness for up to three months, when it was necessary a second infiltration. In this way, there was also an improvement of the values of the Barthel Index Scale, Adductor Tone Rating Scale and Hygiene Score. Good values of muscular properties have an important repercussion on the rehabilitative treatment[22], enabling a better mobilization of the lower limbs and preventing the establishment of muscular contractures and articular deformities, treating with surgical technique[23]; all this with an important impact on the quality of the life of the patient.

\section{CONCLUSIONS}

Our preliminary study shows the possibility to use BTXA in the treatment of the spasticity in patients with ALS no responders to oral antispastic drugs. In the near future it is planned the monitoring of patients clinically and with miometric measure after the following infiltrations. We also planned to increase number of patients to obtain more informations about the efficacy of BTX-A in spasticity of these patients.

\section{CONFLICT OF INTEREST}

The authors confirm that this article content has no conflict of interest.

\section{ACKNOWLEDGEMENTS}

Declared none.

\section{REFERENCES}

[1] Morfini GA, Bosco DA, Brown H, Gatto R, Kaminska A, Song Y, Molla L, Baker L, Marangoni MN, Berth S, Tavassoli E, Bagnato C, Tiwari A, Hayward LJ, Pigino GF, Watterson DM, Huang CF, Banker G, Brown RH Jr, Brady ST. Inhibition of Fast Axonal Transport by Pathogenic SOD1 Involves Activation of p38 MAP Kinase. PLoS One. 2013 Jun 12;8(6):e65235.

[2] Ruth Alonso, Diana Pisa, Ana Isabel Marina, Esperanza Morato, Alberto Rábano, Izaskun Rodal, Luis Carrasco. Evidence for Fungal Infection in Cerebrospinal Fluid and Brain Tissue from Patients with Amyotrophic Lateral Sclerosis. Int J Biol Sci 2015; 11(5):546-558.

[3] Mitchell JD, Wokke JH, Borasio GD. Recombinant human insulinlike growth factor I (rhIGF-I) for amyotrophic lateral sclerosis/motor neuron disease. The Cochrane database of systematic reviews. 2007:CD002064.

[4] Brooks BR, Miller RG, Swash M, Munsat TL. El Escorial revisited: revised criteria for the diagnosis of amyotrophic lateral sclerosis. Amyotroph Lateral Scler Other Motor Neuron Disord. 2000 Dec;1(5):293-9.

[5] Welsh RC, Jelsone-Swain LM, Foerster BR. The utility of independent component analysis and machine learning in the identification of the amyotrophic lateral sclerosis diseased brain. Front Hum Neurosci. 2013 Jun 10;7:251.

[6] He F, Balling R. The role of regulatory T cells in neurodegenerative diseases. Wiley interdisciplinary reviews. 2013;5:153-80.
[7] Engelhardt JI, Tajti J, Appel SH. Lymphocytic infiltrates in the spinal cord in amyotrophic lateral sclerosis. Archives of neurology. 1993;50:30-6.

[8] Grammas P. Neurovascular dysfunction, inflammation and endothelial activation: implications for the pathogenesis of Alzheimer's disease. Journal of neuroinflammation. 2011;8:26.

[9] Mantovani S, Garbelli S, Pasini A, Alimonti D, Perotti C, Melazzini M. et al. Immune system alterations in sporadic amyotrophic lateral sclerosis patients suggest an ongoing neuroinflammatory process. Journal of neuroimmunology. 2009;210:73-9.

[10] Jung HH, Neumann M, Bloch KE. Amyotrophic lateral sclerosis-diagnosis and treatment. Praxis (Bern 1994). 2012 Jul 4;101(14):907-14.

[11] Larbi A, Pawelec G, Witkowski JM, Schipper HM, Derhovanessian E, Goldeck D. et al. Dramatic shifts in circulating CD4 but not CD8 T cell subsets in mild Alzheimer's disease. J Alzheimers Dis. 2009; 17:91-103.

[12] Rentzos M, Evangelopoulos E, Sereti E, Zouvelou V, Marmara S, Alexakis T. et al. Alterations of T cell subsets in ALS: a systemic immune activation? Acta neurologica Scandinavica. 2011;125:260-4.

[13] Saresella M, Calabrese E, Marventano I, Piancone F, Gatti A, Calvo MG. et al. $P D 1$ negative and $P D 1$ positive $C D 4+T$ regulatory cells in mild cognitive impairment and Alzheimer's disease. J Alzheimers Dis. 2010;21:927-38.

[14] Kinsley L, Siddique. Amyotrophic Lateral Sclerosis Overview. T.Editors In: Pagon RA, Adam MP, Ardinger HH, Wallace SE, Amemiya A, Bean LJH, Bird TD, Fong CT, Mefford HC, Smith RJH, Stephens K, editors. Source GeneReviews ${ }^{\circledR}$ [Internet]. Seattle (WA): University of Washington, Seattle. 2001 Mar 23 [updated 2015 Feb 12].

[15] Ashworth NL1, Satkunam LE, Deforge D. Treatment for spasticity in amyotrophic lateral sclerosis/motor neuron disease. Cochrane Database Syst Rev. 2012 Feb 15;(2):CD004156.

[16] Ianieri G, Saggini R, Marvulli R, Tondi G, Aprile A, Ranieri M, Benedetto G, Altini S, Lancioni GE, Goffredo L, Bellomo RG, Megna M, Megna G. New approach in the assessment of the tone, elasticity and the muscular resistance: nominal scales vs MYOTON. Int J Immunopathol Pharmacol. 2009 Jul-Sep;22(3 Suppl):21-4.

[17] Chuang LL, Wu CY, Lin KC, Lur SY. Quantitative mechanical properties of the relaxed biceps and triceps brachii muscles in patients with subacute stroke: a reliability study of the myoton-3 myometer. Stroke Res Treat. 2012;2012:617694. Epub 2012 Apr 30.

[18] McElhiney MC, Rabkin JG, Gordon PH, Goetz R, Mitsumoto H Prevalence of fatigue and depression in ALS patients and change over time. J Neurol Neurosurg Psychiatry. 2009 Oct;80(10):1146-9.

[19] Squires N, Humberstone M, Wills A, Arthur A. The use of botulinum toxin injections to manage drooling in amyotrophic lateral sclerosis/motor neurone disease: a systematic review. Dysphagia. 2014 Aug;29(4):500-8. Epub 2014 May 22.

[20] Lui AJ, Byl NN. A systematic review of the effect of moderate intensity exercise on function and disease progression in amyotrophic lateral sclerosis. J Neurol Phys Ther. 2009 Jun;33(2):6887.

[21] Lo Coco D, La Bella V. Fatigue, sleep, and nocturnal complaints in patients with amyotrophic lateral sclerosis. Eur J Neurol. 2012 May;19(5):760-3. Epub 2012 Jan 10.

[22] Smania N, Picelli A, Munari D, Geroin C, Ianes P, Waldner A, Gandolfi M. Rehabilitation procedures in the management of spasticity Eur J Phys Rehabil Med. 2010 Sep;46(3):423-38.

[23] de Moraes Barros Fucs PM, Svartman C, de Assumpção RM, Yamada HH, Simis SD. Surgical technique: Medial column arthrodesis in rigid spastic planovalgus feet. Clin Orthop Relat Res. 2012 May;470(5):1334-43. 